\title{
RESONANCE AND QUASILINEAR ELLIPTICITY
}

BY

\author{
VICTOR L. SHAPIRO
}

\begin{abstract}
Two resonance-type existence theorems for periodic solutions of second order quasilinear elliptic partial differential equations are established. The first theorem is a best possible result, and the second theorem presents conditions which are both necessary and sufficient.
\end{abstract}

1. Introduction. In this paper, we establish two resonance-type existence theorems for periodic solutions of second order quasilinear elliptic partial differential equations. The first theorem is a best possible result, and the second theorem presents conditions which are both necessary and sufficient. Also, we shall allow both the higher and lower order coefficients of the partial differential equation considered to be unbounded. There are few (if any) papers in the literature which deal with results of this nature, i.e., incorporating quasilinearity and unboundedness in both the higher order terms and lower order terms, all at resonance.

Let $\Omega=\left\{x:-\pi<x_{j}<\pi, j=1, \ldots, N\right\}$ be the $N$-torus, $N \geqslant 2 . \phi \in C^{\infty}(\Omega)$ means that $\phi \in C^{\infty}\left(\mathbf{R}^{N}\right)$ and is periodic of period $2 \pi$ in each variable. $W^{1,2}(\Omega)$ will designate the familiar closure with respect to these $C^{\infty}$, periodic functions (that is, with respect to $C^{\infty}(\Omega)$ ).

We shall consider second order, quasilinear elliptic operators $Q$, operating on $W^{1,2}(\Omega)$, of the form

$$
Q u=-D_{i}\left[a^{i j}(x, u) D_{j} u\right]+b^{j}(x, u, D u) D_{j} u,
$$

where $D u$ represents the gradient of $u, D_{j} u=\partial u / \partial x_{j}$, and the summation convention is employed for $i, j=1, \ldots, N$.

The coefficients of $Q$, namely, the functions $a^{i j}(x, z)$ and $b^{j}(x, z, p)$, are assumed to be defined for all values of $(x, z) \in(\Omega-E) \times \mathbf{R}$ and $(x, z, p) \in(\Omega-E) \times \mathbf{R}$ $\times \mathbf{R}^{N}$, respectively, where $E \subset \Omega$ is a set of Lebesgue measure zero. Furthermore, we shall suppose the following throughout the paper.

(Q-1) The coefficients $a^{i j}(x, z)$ and $b^{j}(x, z, p)$ satisfy the usual Carathéodory conditions: For each fixed $z \in \mathbf{R}$ and $p \in \mathbf{R}^{N}$, the functions $a^{i j}(x, z)$ and $b^{j}(x, z, p)$ are measurable; for a.e. $x \in \Omega$ the functions $a^{i j}(x, z)$ and $b^{j}(x, z, p)$ are respectively continuous in $\mathbf{R}$ and $\mathbf{R} \times \mathbf{R}^{N}, i, j=1, \ldots, N$.

(Q-2) $\exists$ a nonnegative function $a(x) \in L^{2}(\Omega)$ such that $\left|a^{i j}(x, z)\right| \leqslant a(x)$ for every $z \in \mathbf{R}$ and a.e. $x \in \Omega, i, j=1, \ldots, N$.

Received by the editors October 19, 1984 and, in revised form, April 20, 1985.

1980 Mathematics Subject Classification. Primary 35J60; Secondary 35D05.

Key words and phrases. Quasilinear elliptic, resonance, distribution solution, unbounded coefficients.

(c1986 American Mathematical Society $0002-9947 / 86 \$ 1.00+\$ .25$ per page 
(Q-3) $Q$ is symmetric; that is, $a^{i j}(x, z)=a^{j i}(x, z)$ for every $z \in \mathbf{R}$ and a.e. $x \in \Omega, i, j=1, \ldots, N$.

(Q-4) $Q$ is uniformly elliptic almost everywhere in $\Omega$; that is, there is a constant $\eta_{0}>0$ such that

$$
a^{i j}(x, z) \xi_{i} \xi_{j} \geqslant \eta_{0}|\xi|^{2}
$$

for every $z \in \mathbf{R}$, a.e. $x \in \Omega$, and every $\xi \in \mathbf{R}^{N}\left(|\xi|^{2}=\xi_{1}^{2}+\cdots+\xi_{N}^{2}\right)$.

(Q-5) There is a nonnegative function $b(x) \in L^{2}(\Omega)$ and positive constants $\eta_{1}$ and $\eta_{2}$ such that

$$
\left|b^{j}(x, z, p)\right| \leqq b(x)+\eta_{1}|z|+\eta_{2}|p|
$$

for every $p \in \mathbf{R}^{N}, z \in \mathbf{R}$, and a.e. $x \in \Omega, j=1, \ldots, N$.

(Q-6) For every $u \in W^{1,2}(\Omega)$, the vector

$$
\mathbf{b}(x, u, D u)=\left[b^{1}(x, u, D u), \ldots, b^{N}(x, u, D u)\right]
$$

is weakly solenoidal, i.e., $\int_{\Omega} b^{j}(x, u, D u) D_{j} v(x) d x=0$ for every $u$ and $v \in W^{1,2}(\Omega)$ where the summation convention is used.

(Q-7) If $\left\{u^{n}\right\}_{n=1}^{\infty}$ is a sequence of functions in $L^{2}(\Omega)$ which tend strongly to $u \in L^{2}(\Omega)$ and $\left\{\mathbf{w}^{n}\right\}_{n=1}^{\infty}$ is a sequence of vector-valued functions which tend weakly to $\mathbf{w} \in\left[L^{2}(\Omega)\right]^{N}$, then $\left\{\mathbf{b}\left(x, u^{n}, \mathbf{w}^{n}\right)\right\}_{n=1}^{\infty}$ tends weakly to $\mathbf{b}(x, u, \mathbf{w}) \in\left[L^{2}(\Omega)\right]^{N}$, i.e., with $b^{j}(x, u, \mathbf{w}) \equiv b^{j}\left(x, u, w_{1}, \ldots, w_{n}\right)$ the $j$ th component of $\mathbf{b}(x, u, \mathbf{w})$, then $\lim _{n \rightarrow \infty} \int_{\Omega} b^{j}\left(x, u^{n}, \mathbf{w}^{n}\right) v d x=\int_{\Omega} b^{j}(x, u, w) v d x \forall v \in L^{2}(\Omega)$ and $j=1, \ldots, N$.

By strong convergence in (Q-7), we mean convergence in norm.

Before proceeding, we give an example of a vector $\mathbf{b}(x, z, p)$ which meets $(\mathrm{Q}-5)$, (Q-6), and (Q-7). Define the $j$ th component of $\mathbf{b}(x, z, p)$ as follows

$$
\begin{array}{ll}
b^{1}(x, z, p)=\cos x_{2}+p_{2} \sin x_{1}, & b^{2}(x, z, p)=\cos x_{1}-p_{1} \sin x_{1}-z \cos x_{1}, \\
b^{j}(x, z, p) \equiv 0 \text { for } j=3, \ldots, N .
\end{array}
$$

Clearly, condition (Q-5) is met. If $\phi \in C^{\infty}(\Omega)$, then

$$
\int_{\Omega} \cos x_{2} D_{1} \phi(x) d x=\int_{\Omega} \cos x_{1} D_{2} \phi(x)=0 .
$$

Likewise if $u \in W^{1,2}(\Omega)$, then

$$
\begin{aligned}
& \int_{\Omega}[\left.D_{2}\left(u \sin x_{1}\right) D_{1} \phi-D_{1}\left(u \sin x_{1}\right) D_{2} \phi\right] d x \\
&=-\int_{\Omega} u \sin x_{1}\left[D_{2} D_{1} \phi-D_{1} D_{2} \phi\right] d x=0 ;
\end{aligned}
$$

so, it is clear that (Q-6) is met. To see that (Q-7) is met, let $\left\{u^{n}\right\}_{n=1}^{\infty}$ tend strongly to $u$ in $L^{2}(\Omega)$ and let $\left\{\mathbf{w}^{n}\right\}$ tend weakly to $\mathbf{w} \in\left[L^{2}(\Omega)\right]^{N}$. Then $b^{1}\left(x, u^{n}, \mathbf{w}^{n}\right)=\cos x_{2}$ $+w_{2}^{n} \sin x_{1}$ and $b^{2}\left(x, u^{n}, \mathbf{w}^{n}\right)=\cos x_{1}-w_{1}^{n} \sin x_{1}-u^{n} \cos x_{1}$ and $b_{j}\left(x, u^{n}, \mathbf{w}^{n}\right)=0$ for $j=3, \ldots, N$. It is clear that

$$
\lim _{n \rightarrow \infty} \int_{\Omega} b^{j}\left(x, u^{n}, \mathbf{w}^{n}\right) v d x=\int_{\Omega} b^{j}(x, u, \mathbf{w}) v d x \quad \forall v \in L^{2}(\Omega),
$$

for $j=1, \ldots, N$. Consequently condition (Q-7) is met. Therefore $\mathbf{b}(x, z, p)$ has met all the asserted conditions and our example is complete. 
We shall study equations of the form

$$
Q u=f(x, u)
$$

under various assumptions on $f(x, u)$. Let $\mathscr{N} u$ be the Nemitsky operator

$$
\mathscr{N} u=f(x, u(x))
$$

where $f(x, z): \Omega \times \mathbf{R} \rightarrow \mathbf{R}$. We shall suppose throughout the paper that

(f-1) $f(x, z)$ meets the same Carathéodory conditions that $a^{i j}(x, z)$ meets in (Q-1) above.

(f-2) For each $r>0$, there is a $\zeta_{r} \in L^{2}(\Omega)$ such that $|f(x, z)| \leqslant \zeta_{r}(x)$ for a.e. $x \in \Omega$ and $|z| \leqslant r$.

To establish the first theorem in the paper, we shall need the following one-sided growth condition on $f$ :

(f-3) Given $\varepsilon>0, \exists$ a nonnegative function $c_{\varepsilon}(x)$ in $L^{2}(\Omega)$ and a constant $z_{0}(\varepsilon)$,

$$
z f(x, z) \leqslant \varepsilon z^{2}+c_{\varepsilon}(x)|z| \text { for }|z| \geqslant z_{0}(\varepsilon) \text { and a.e. } x \in \Omega .
$$

We note that (f-3) is a generalization of the notion $\limsup _{|z| \rightarrow \infty} f(x, z) / z \leqslant 0$ uniformly for $x \in \Omega$.

Motivated by [3], we shall set

$$
\mathscr{F}_{ \pm}(x)=\limsup _{z \rightarrow \pm \infty} f(x, z) / z
$$

and establish the following theorem in $\S 3$.

Theorem 1. Assume (Q-1)-(Q-7), (f-1), (f-2), and (f-3). Suppose

(i) $\int_{\Omega} \mathscr{F}_{+}(x) d x<0$ and $\int_{\Omega} \mathscr{F}_{-}(x) d x<0$,

where $\mathscr{F}_{+}$and $\mathscr{F}_{-}$are defined in (1.3). Then there exists a distribution solution $u \in W^{1,2}(\Omega)$ of $Q u=f(x, u)$ with $f(x, u) \in L^{1}(\Omega)$ and $f(x, u) u \in L^{1}(\Omega)$.

To be quite explicit, what we mean by $u \in W^{1,2}(\Omega)$ being a distribution solution of $Q u=f(x, u)$ is

$$
(1.3)^{\prime} \quad \int_{\Omega}\left[a^{i j}(x, u) D_{j} u D_{i} \phi d x+\phi b^{j}(x, u, D u) D_{j} u\right] d x=\int_{\Omega} f(x, u) \phi d x
$$

for every $\phi \in C^{\infty}(\Omega)$, where the summation convention is used for $i, j=1, \ldots, N$.

Theorem 1 is in a certain sense a best possible result, i.e., if we replace (i) above by $\left(\mathrm{i}^{\prime}\right)$ or $\left(\mathrm{i}^{\prime \prime}\right)$ below, the conclusion to the theorem is false.

(i') $\int_{\Omega} \mathscr{F}_{+}(x) d x=0$ and $\int_{\Omega} \mathscr{F}_{-}(x) d x<0$.

(i") $\int_{\Omega} \mathscr{F}_{+}(x) d x<0$ and $\int_{\Omega} \mathscr{F}_{-}(x) d x=0$.

To see that this is the case for (i'), we shall suppose Theorem 1 holds if (i) is replaced by (i') and arrive at a contradiction. Set

$$
\beta(z)= \begin{cases}0, & z \geqslant 0 \\ -z, & z<0\end{cases}
$$

and $f(x, z)=1+\beta(z)$ for $x \in \Omega$ and $z \in \mathbf{R}$. Then $f(x, z)$ meets (f-1), (f-2), and (f-3). Furthermore, it follows from (1.3) and (1.4) that $\mathscr{F}_{+}(x)=0$ and $\mathscr{F}_{-}(x)=-1$ for $x \in \Omega$, and therefore condition (i') is met. So if Theorem 1 were true with (i) replaced by (i'), a distribution solution $u \in W^{1,2}(\Omega)$ of $Q u=f(x, u)$ would exist; i.e., we would have that $(1.3)^{\prime}$ holds. 
On setting $\phi=1$ in $(1.3)^{\prime}$ and observing that $D_{i} 1=0$ for $i=1, \ldots, N$, we obtain in particular from $(1.3)^{\prime}$ that

$$
\int_{\Omega} b^{j}(x, u, D u) D_{j} u=\int_{\Omega} f(x, u) d x,
$$

where the summation convention is used for $j=1, \ldots, N$.

Now $f(x, u)=1+\beta[u(x)]$. So it is easily seen from (1.4) that

$$
f(x, u(x)) \geqslant 1 \text { a.e. in } \Omega \text {. }
$$

Also we observe from (Q-6) that the left-hand side of (1.5) is 0 . We consequently obtain from (1.5) and (1.6) that $0 \geqslant(2 \pi)^{N}$, a contradiction. Therefore no distribution solution $u \in W^{1,2}(\Omega)$ satisfying (1.3)' exists. We conclude that Theorem 1 does not hold when (i) is replaced by (i'). An analogous example shows that Theorem 1 does not hold when (i) is replaced by $\left(i^{\prime \prime}\right)$. Theorem 1 is indeed in a certain sense a best possible result.

Theorem 2 deals with $f(x, u)$ in the form $g(u)-h$, i.e., with

$$
Q u=g(u)-h \text {. }
$$

In (1.7), $h$ is in $W^{-1,2}(\Omega)\left(\equiv W^{1,2}(\Omega)^{*}\right)$. To be quite specific, $h: W^{1,2}(\Omega) \rightarrow \mathbf{R}$, and $h$ is both linear and continuous. On the other hand, $g$ will be in $C(\mathbf{R})$ and we shall suppose that both $\operatorname{limits}_{\lim _{z \rightarrow \infty}} g(z)$ and $\lim _{z \rightarrow-\infty} g(z)$ exist and are finite. We shall say that $u \in W^{1,2}(\Omega)$ is a distribution solution of (1.7) if the following prevails:

$$
\int_{\Omega}\left[a^{i j}(x, u) D_{j} u D_{i} \phi+\phi b^{j}(x, u, D u) D_{j} u\right] d x=\int_{\Omega} g(u) \phi d x-h(\phi)
$$

for every $\phi \in C^{\infty}(\Omega)$.

The theorem we shall prove is the following.

Theorem 2. Assume (Q-1)-(Q-7), that $g \in C(\mathbf{R}), h \in W^{-1,2}(\Omega)$, and that the limits $\lim _{z \rightarrow \infty} g(z)=g(\infty)$ and $\lim _{z \rightarrow-\infty} g(z)=g(-\infty)$ exist and are finite. Suppose also that

$$
g(\infty)<g(z)<g(-\infty) \quad \text { for } z \in \mathbf{R} .
$$

Then a necessary and sufficient condition that a distribution solution $u \in W^{1,2}(\Omega)$ of $Q u=g(u)-h$ exists is that

$$
(2 \pi)^{N} g(\infty)<h(1)<(2 \pi)^{N} g(-\infty) .
$$

The results stated in this paper were motivated by the well-known results of Brezis and Nirenberg [2, Chapter IV], De Figueiredo and Gossez [3], Hess [4], and Landesman and Lazer [6].

We observe that $\psi \equiv 1$ is a distribution solution of (1.2) with $f(x, z) \equiv 0$, i.e.,

$$
\int_{\Omega}\left[a^{i j}(x, \psi) D_{j} \psi D_{i} \phi+\phi b^{j}(x, \psi, D \psi) D_{j} \psi\right] d x=0
$$

for every $\phi \in C^{\infty}(\Omega)$. Consequently, we see that $Q(\psi)=0 \psi$ and therefore that 0 is a characteristic value. We can therefore refer to our existence theorems involving (1.2) and (1.7) as resonance-type theorems (see the discussion in [3]). 
From Theorem 1, we can also easily obtain the following result.

Corollary. Assume (Q-1)-(Q-7), (f-1), and (f-2). Suppose that

$$
\limsup _{|z| \rightarrow \infty} f(x, z) / z<0 \quad \text { uniformly for } x \in \Omega \text {. }
$$

Then there exists a distribution solution of $Q u=f(x, u)$ with $f(x, u) \in L^{1}(\Omega)$.

(1.11) and (1.3) imply that $f$ meets (f-3) and (i) of Theorem 1. Therefore the corollary is an immediate consequence of Theorem 1.

An interesting fact about the above corollary is that it constitutes a partial generalization of an important result in the well-known paper of Kazdan and Warner, namely, part (b) of Corollary 2.12 [5, p. 575].

Even though we have not done so here, the techniques used in this paper allow us to establish results for our quasilinear $Q$ analogous to those established in [3, Theorem 4, p. 13 and 2, pp. 286-288]. We shall treat results of this nature and related matters in a sequel to this current paper.

2. Fundamental lemmas. We shall use a Galerkin technique to establish our existence theorems. In order to accomplish this, we observe that there is a sequence $\left\{\psi_{k}\right\}_{k=1}^{\infty}$ of real-valued functions in $C^{\infty}(\Omega)$ with the following properties:

(a) $\psi_{1}=(2 \pi)^{-N / 2}$,

(b) $\int_{\Omega} \psi_{k} \psi_{l} d x=\delta_{k l}$ where $\delta_{k l}$ is the Kronecker delta, $k, l=1,2, \ldots$.

Also, given $\psi \in C^{\infty}(\Omega)$ and $\varepsilon>0, \exists$ constants $c_{1}, \ldots, c_{n}$ such that

$$
\left|\psi(x)-c_{q} \psi_{q}(x)\right|<\varepsilon \text { and }\left|D_{j} \psi(x)-c_{q} D_{j} \psi_{q}(x)\right|<\varepsilon
$$

uniformly for $x \in \Omega$ and $j=1, \ldots, N$, where the summation convention is used in (2.2) with $q=1, \ldots, n$.

The first lemma we prove is the following, where $\left\{\psi_{k}\right\}_{k=1}^{\infty}$ is the sequence in (2.1) and (2.2).

LEMMA 1. Let $F(x)$ be a nonnegative function in $L^{1}(\Omega)$ and let $f(x, z)$ satisfy (f-1) and (f-2). Suppose that $|f(x, z)| \leqslant F(x)$ for $z \in \mathbf{R}$ and a.e. $x \in \Omega$ and that $Q$ satisfies (Q-1)-(Q-6). Then if $n$ is a given positive integer, there is a function $u=\gamma_{1} \psi_{1}+\cdots+\gamma_{n} \psi_{n}$ such that

$$
\begin{gathered}
\int_{\Omega}\left[a^{i j}(x, u) D_{j} u D_{i} \psi_{k}+\psi_{k} b^{j}(x, u, D u) D_{j} u+u \psi_{k} n^{-1}\right] d x \\
\quad=\int_{\Omega} \psi_{k}(x) f(x, u) d x
\end{gathered}
$$

for $k=1, \ldots, n$, where the summation convention is used for $i, j=1, \ldots, N$.

Here, and throughout the rest of the paper, we shall use the notation

$$
\langle v, w\rangle_{0}=\int_{\Omega} v(x) w(x) d x
$$

for $v$ and $w$ in $L^{2}(\Omega)$. 
For each $\alpha=\left(\alpha_{1}, \ldots, \alpha_{n}\right) \in \mathbf{R}^{n}$, we introduce an $n \times n$ matrix $A(\alpha)$ with components $A_{k l}(\alpha)$ given as follows:

$$
\begin{aligned}
A_{k l}= & \left\langle D_{i} \psi_{k}, a^{i j}\left(\cdot, \alpha_{q} \psi_{q}\right) D_{j} \psi_{l}\right\rangle_{0} \\
& +\left\langle\psi_{k}, b^{j}\left(\cdot, \alpha_{q} \psi_{q}, D \alpha_{q} \psi_{q}\right) D_{j} \psi_{l}\right\rangle_{0}+\left\langle\psi_{k}, \psi_{l}\right\rangle_{0} n^{-1},
\end{aligned}
$$

where the summation convention is used with indices $i, j=1, \ldots, N$ and $q=$ $1, \ldots, n$.

We observe from (Q-1), (Q-2), (Q-5), and (2.4) that

$$
A_{k l}(\alpha) \in C\left(\mathbf{R}^{n}\right) \text { for } k, l=1, \ldots, n .
$$

Next, we observe from (2.5) for $\beta=\left(\beta_{1}, \ldots, \beta_{n}\right)$,

$$
\begin{aligned}
A_{k l}(\alpha) \beta_{l}= & \left\langle D_{i} \psi_{k}, a^{i j}\left(\cdot, \alpha_{q} \psi_{q}\right) D_{j} \beta_{l} \psi_{l}\right\rangle_{0} \\
& +\left\langle\psi_{k}, b^{j}\left(\cdot, \alpha_{q} \psi_{q}, D \alpha_{q} \psi_{q}\right) D_{j} \beta_{l} \psi_{l}\right\rangle_{0}+\left\langle\psi_{k}, \beta_{l} \psi_{l}\right\rangle_{0} n^{-1}
\end{aligned}
$$

Now it follows from (Q-6), the fact that $D_{j} \psi^{2}=2 \psi D_{j} \psi$, and from (2.4) that

$$
\left\langle\psi, b^{j}(\cdot, \phi, D \phi) D_{j} \psi\right\rangle_{0}=0
$$

for $\phi$ and $\psi \in C^{\infty}(\Omega)$. Consequently, we see from (2.7) that the quadratic form $\beta \cdot A(\alpha) \beta=\beta_{k} A_{k l}(\alpha) \beta_{l}$ is such that

$$
\beta \cdot A(\alpha) \beta=\left\langle D_{i} \psi, a^{i j}\left(\cdot, \alpha_{q} \psi_{q}\right) D_{j} \psi\right\rangle_{0}+\langle\psi, \psi\rangle_{0} n^{-1},
$$

where $\psi=\beta_{1} \psi_{1}+\cdots+\beta_{n} \psi_{n}$.

It follows from (Q-4) and (2.4) that the right-hand side of (2.8) majorizes

$$
\eta_{0} \int_{\Omega}|D \psi|^{2} d x+\langle\psi, \psi\rangle_{0} n^{-1}
$$

where $\psi=\beta_{1} \psi_{1}+\cdots+\beta_{n} \psi_{n}$ and $|D \psi|^{2}=\left|D_{1} \psi\right|^{2}+\cdots+\left|D_{n} \psi\right|^{2}$. We conclude therefore from (2.8) and (2.1)(b) that

$$
\beta \cdot A(\alpha) \beta \equiv \beta_{k} A_{k l}(\alpha) \beta_{l} \geqslant|\beta|^{2} n^{-1} \text {. }
$$

Since $\beta$ is arbitrary in $\mathbf{R}^{n}$, it follows from (2.9) that for each $\alpha \in \mathbf{R}^{n}$, the inverse matrix $[A(\alpha)]^{-1}$ exists, and furthermore

$$
\left\|[A(\alpha)]^{-1}\right\|_{\mathscr{M}} \leqslant n \quad \forall \alpha \in \mathbf{R}^{n}
$$

where $\|\cdot\|_{\mathscr{M}}$ designates the usual $n \times n$ matrix norm.

Next, for each $\alpha \in \mathbf{R}^{n}$, we set

$$
S_{k}(\alpha)=\left\langle\psi_{k}, f\left(\cdot, \alpha_{q} \psi_{q}\right)\right\rangle_{0}
$$

for $k=1, \ldots, n$, where the summation convention is used for $q=1, \ldots, n$. We observe from (f-1), (f-2), and (2.4) that $S_{k}(\alpha) \in C\left(\mathbf{R}^{n}\right)$ for $k=1, \ldots, n$. Also, we see from (2.11) and from the fact that $|f(x, z)| \leqslant F(x)$ for a.e. $x \in \Omega$ and every $z \in \mathbf{R}$ where $F \in L^{1}(\Omega)$ that there is a constant $\Gamma_{1}$ such that $\left|S_{k}(\alpha)\right| \leqslant \Gamma_{1}$ for every $\alpha \in \mathbf{R}^{n}, k=1, \ldots, n$. We set

$$
S(\alpha)=\left(S_{1}(\alpha), \ldots, S_{n}(\alpha)\right),
$$


and conclude that

$S$ is a continuous map of $\mathbf{R}^{n}$ into the closed ball of $\mathbf{R}^{n}$ with center 0 and radius $n^{1 / 2} \Gamma_{1}$.

Next, we set $G(\alpha)=[A(\alpha)]^{-1} S(\alpha)$ and observe from (2.6), (2.10), and (2.12)(a), (b) that $G(\alpha)$ is a continuous function of $\alpha$ which maps $\mathbf{R}^{n}$ into the closed ball of $\mathbf{R}^{n}$ with center 0 and radius $n^{3 / 2} \Gamma_{1}$. Consequently, $G$ is a continuous map of this last mentioned closed ball into itself. We invoke the Brouwer fixed point theorem and conclude that there exists $\gamma=\left(\gamma_{1}, \ldots, \gamma_{n}\right) \in \mathbf{R}^{n}$ such that $[A(\gamma)]^{-1} S(\gamma)=\gamma$, i.e., $A(\gamma) \gamma=S(\gamma)$. We set $u=\gamma_{1} \psi_{1}+\cdots+\gamma_{n} \psi_{n}$ and obtain from (2.7), (2.11), and (2.12)(a), with $\alpha=\beta=\gamma$, that

$$
\left\langle D_{i} \psi_{k}, a^{i j}(\cdot, u) D_{j} u\right\rangle_{0}+\left\langle\psi_{k}, b^{j}(\cdot, u, D u) D_{j} u\right\rangle_{0}+\left\langle\psi_{k}, u\right\rangle_{0} n^{-1}=\left\langle\psi_{k}, f(\cdot, u)\right\rangle_{0}
$$

for $k=1, \ldots, n$. But this is (2.3) in the statement of the lemma, and the proof of the lemma is complete.

The next lemma we prove is

LEMMA 2. Let $n$ be a given positive integer and let $f(x, z)$ satisfy (f-1) and (f-2). Suppose that $Q$ satisfies (Q-1)-(Q-6) and that there is a nonnegative function $F(x)$ in $L^{2}(\Omega)$ such that

$$
z f(x, z) \leqslant F(x)|z|+z^{2} / 2 n \text { for a.e. } x \in \Omega \text { and } \forall z \in \mathbf{R} .
$$

Then there is a function $u=\gamma_{1} \psi_{1}+\cdots+\gamma_{n} \psi_{n}$ such that (2.3) in Lemma 1 holds.

For each positive integer $M$ set

$$
f^{M}(x, z)= \begin{cases}f(x, M), & z \geqslant M, \\ f(x, z), & -M \leqslant z \leqslant M, \\ f(x,-M), & z \leqslant-M .\end{cases}
$$

It follows from (f-2) that there is a $\zeta_{M} \in L^{2}(\Omega)$ such that

$$
\left|f^{M}(x, z)\right| \leqslant \zeta_{M}(x) \text { for a.e. } x \in \Omega \text { and } \forall z \in \mathbf{R} \text {. }
$$

Consequently it follows from Lemma 1 that there exists $\left\{\gamma_{i}^{M}\right\}_{i=1}^{n}$ such that

$$
u^{M}=\gamma_{1}^{M} \psi_{1}+\cdots+\gamma_{n}^{M} \psi_{n}
$$

satisfies (2.3) with $f$ replaced by $f^{M}$, i.e.,

$$
\begin{aligned}
\left\langle D_{i} \psi_{k}, a^{i j}\left(\cdot, u^{M}\right) D_{j} u^{M}\right\rangle_{0}+\left\langle\psi_{k}, b^{j}\left(\cdot, u^{M}, D u^{M}\right) D_{j} u^{M}\right\rangle_{0}+\left\langle\psi_{k}, u^{M}\right\rangle n^{-1} & \\
& =\left\langle\psi_{k}, f^{M}\left(\cdot, u^{M}\right)\right\rangle_{0}
\end{aligned}
$$

for $k=1, \ldots, n$.

Now it follows from the definition of $f^{M}(x, z)$ and from (2.13) that $z f^{M}(x, z) \leqslant$ $F(x)|z|+|z|^{2} / 2 n$ for a.e. $x \in \Omega$ and $\forall z \in \mathbf{R}$. Consequently

$$
u^{M}(x) f^{M}\left[x, u^{M}(x)\right] \leqslant\left|u^{M}(x)\right| F(x)+\left|u^{M}(x)\right|^{2} / 2 n
$$

for a.e. $x \in \Omega$ and $M=1,2, \ldots$. 
Next, we multiply both sides of (2.15) by $\gamma_{k}^{M}$ and sum over $k=1, \ldots, n$. Using (Q-4), (2.7)', and (2.14), we then obtain that

$$
n^{-1}\left\langle u^{M}, u^{M}\right\rangle_{0} \leqslant\left\langle u^{M}, f^{M}\left(\cdot, u^{M}\right)\right\rangle_{0}
$$

for each fixed positive integer $M$. But then it follows from (2.16) that

$$
(2 n)^{-1}\left\langle u^{M}, u^{M}\right\rangle_{0} \leqslant \int_{\Omega}\left|u^{M}(x)\right| F(x) d x .
$$

By the hypothesis of the lemma, $F \in L^{2}(\Omega)$; so it follows from this last inequality in conjunction with the Schwarz inequality that

$$
\left\langle u^{M}, u^{M}\right\rangle_{0} \leqslant 4 n^{2}\langle F, F\rangle_{0} .
$$

We next employ (2.1)(b) and (2.14) in conjunction with this last fact to obtain

$$
\left(\gamma_{1}^{M}\right)^{2}+\cdots+\left(\gamma_{n}^{M}\right)^{2} \leqslant 4 n^{2}\langle F, F\rangle_{0}
$$

for every positive integer $M$.

Since $n$ is a fixed positive integer, we infer from this last inequality that there exists a subsequence of $\left\{\gamma_{k}^{M}\right\}$ which converges for each $k=1, \ldots, n$. For ease of notation, we shall suppose this subsequence is the full sequence and record this fact as

$$
\lim _{M \rightarrow \infty} \gamma_{k}^{M}=\gamma_{k}, \quad k=1, \ldots, n .
$$

We set $u=\gamma_{1} \psi_{1}+\cdots+\gamma_{n} \psi_{n}$ and obtain from (2.14) and (2.17) that

$$
\begin{aligned}
& \lim _{M \rightarrow \infty} u^{M}(x)=u(x) \quad \text { uniformly for } x \in \Omega, \\
& \lim _{M \rightarrow \infty} D_{j} u^{M}(x)=D_{j} u(x) \quad \text { uniformly for } x \in \Omega \text { and } j=1, \ldots, N .
\end{aligned}
$$

From (Q-1) and this last fact, we see that

$$
\begin{aligned}
& \text { (a) } \lim _{M \rightarrow \infty} a^{i j}\left(x, u^{M}(x)\right)=a^{i j}(x, u(x)), \\
& \text { (b) } \lim _{M \rightarrow \infty} b^{j}\left(x, D u^{M}(x)\right)=b^{j}(x, D u(x))
\end{aligned}
$$

for a.e. $x \in \Omega$ and $i, j=1, \ldots, N$.

From (Q-2) in conjunction with (2.14), (2.17), (2.18), and (2.19)(a), we see that

$$
\lim _{M \rightarrow \infty}\left\langle D_{i} \psi_{k}, a^{i j}\left(\cdot, u^{M}\right) D_{j}^{d} u^{M}\right\rangle_{0}=\left\langle D_{i} \psi_{k}, a^{i j}(\cdot, u) D_{j} u\right\rangle_{0}
$$

for $k=1, \ldots, n$. Likewise, we see from (Q-5) in conjunction with (2.14), (2.17), (2.18), and (2.19)(b) that

$$
\lim _{M \rightarrow \infty}\left\langle\psi_{k}, b^{j}\left(\cdot, u^{M}, D u^{M}\right) D_{j} u^{M}\right\rangle_{0}=\left\langle\psi_{k}, b^{j}(\cdot, u, D u) D_{j} u\right\rangle_{0}
$$

for $k=1, \ldots, n$.

Next, we observe from (2.14) and (2.17) that the sequence $\left\{u^{M}(x)\right\}_{M=1}^{\infty}$ is uniformly bounded on $\Omega$ and in $C^{\infty}(\Omega)$ for each $M$. We consequently obtain from the definition of $f^{M}(x, z)$ that there is an $M_{0}$ such that for $M \geqslant M_{0}, f^{M}\left(x, u^{M}(x)\right)$ $=f\left(x, u^{M}(x)\right)$ for $x \in \Omega$. But then it follows from (f-1), (f-2), and (2.18) that

$$
\lim _{M \rightarrow \infty}\left\langle\psi_{k}, f^{M}\left(\cdot, u^{M}\right)\right\rangle_{0}=\left\langle\psi_{k}, f(\cdot, n)\right\rangle_{0}
$$


for $k=1, \ldots, n$. We conclude from (2.15), (2.18), (2.20), (2.21), and this last fact that

$$
\left\langle D_{i} \psi_{k}, a^{i j}(\cdot, u) D_{j} u\right\rangle_{0}+\left\langle\psi_{k}, b^{j}(\cdot, u, D u) D_{j} u\right\rangle_{0}+\left\langle\psi_{k}, u\right\rangle_{0} n^{-1}=\left\langle\psi_{k}, f(\cdot, u)\right\rangle_{0}
$$

for $k=1, \ldots, n$. But this establishes (2.3) for $u=\gamma_{1} \psi_{1}+\cdots+\gamma_{n} \psi_{n}$, and the proof of the lemma is complete.

Next for $w \in L^{2}(\Omega)$, we set

$$
\|w\|_{0}=\left\{\langle w, w\rangle_{0}\right\}^{1 / 2}
$$

and establish the following lemma.

LEMMA 3. Suppose $f(x, z)$ satisfies (f-1), (f-2), and that there is a nonnegative function $F \in L^{2}(\Omega)$ such that

$$
z f(x, z) \leqslant F(x)|z|+z^{2} \text { for a.e. } x \in \Omega \text { and } \forall z \in \mathbf{R} .
$$

Suppose also that for every positive integer, there is $u^{n}=\gamma_{1}^{n} \psi_{1}+\cdots+\gamma_{n}^{n} \psi_{n}$ which satisfies

$$
\begin{aligned}
\left\langle D_{i} \psi_{k}, a^{i j}\left(\cdot, u^{n}\right) D_{j} u^{n}\right\rangle_{0} & +\left\langle\psi_{k}, b^{j}\left(\cdot, u^{n}, D u^{n}\right) D_{j} u^{n}\right\rangle_{0}+\left\langle\psi_{k}, u^{n}\right\rangle_{0} n^{-1} \\
& =\left\langle\psi_{k}, f\left(\cdot, u^{n}\right)\right\rangle_{0}
\end{aligned}
$$

for $k=1, \ldots, n$, where the summation convention is used for $i, j=1, \ldots, N$, and $Q$ satisfies (Q-1)-(Q-6). Suppose furthermore there is a constant $K$ such that

$$
\left\|u^{n}\right\|_{0} \leqslant K \text { for } n=1,2, \ldots
$$

Then there is a constant $K^{*}$ such that

$$
\left\langle\left|f\left(\cdot, u^{n}\right)\right|,\left|u^{n}\right|\right\rangle_{0} \leqslant K^{*} \text { for } n=1,2, \ldots
$$

Multiplying both sides of (2.24) by $\gamma_{k}^{n}$ and summing over $k=1, \ldots, n$, we obtain from the hypothesis of the lemma and $(2.7)^{\prime}$ that

$$
\left\langle D_{i} u^{n}, a^{i j}\left(\cdot, u^{n}\right) D_{j} u^{n}\right\rangle_{0}+\left\langle u^{n}, u^{n}\right\rangle_{0} n^{-1}=\left\langle u^{n}, f\left(\cdot, u^{n}\right)\right\rangle_{0} .
$$

Consequently, we have from $(\mathrm{Q}-4)$ and this last fact that

$$
0 \leqslant\left\langle u^{n}, f\left(\cdot, u^{n}\right)\right\rangle_{0} \text {. }
$$

Next, we set

$$
\begin{aligned}
& A_{n}=\left\{x \in \Omega: u^{n}(x) f\left(x, u^{n}(x)\right) \geqslant 0\right\}, \\
& B_{n}=\left\{x \in \Omega: u^{n}(x) f\left(x, u^{n}(x)\right)<0\right\},
\end{aligned}
$$

and observe from (2.22) and (2.23) that

$$
\int_{A_{n}} u^{n} f\left(x, u^{n}\right) d x \leqslant\|F\|_{0}\left\|u^{n}\right\|_{0}+\left\|u^{n}\right\|_{0}^{2}
$$

for $n=1,2, \ldots$. Consequently we have from (2.25) that there is a constant $K_{1}>0$ such that

$$
\int_{A_{n}} u^{n} f\left(x, u^{n}\right) d x \leqslant K_{1} \quad \text { for } n=1,2, \ldots
$$


Also, it follows from (2.27) and (2.28)

$$
-\int_{B_{n}} u^{n} f\left(x, u^{n}\right) d x \leqslant \int_{A_{n}} u^{n} f\left(x, u^{n}\right) d x .
$$

Therefore, we obtain from (2.29) and this last fact that

$$
\int_{B_{n}}\left|u^{n}\right|\left|f\left(x, u^{n}\right)\right| d x \leqslant K_{1} \quad \text { for } n=1,2, \ldots
$$

But this fact in conjunction with (2.28) and (2.29) gives

$$
\int_{\Omega}\left|u^{n}\right|\left|f\left(x, u^{n}\right)\right| d x \leqslant 2 K_{1} \quad \text { for } n=1,2, \ldots
$$

This last inequality is (2.26) with $K^{*}=2 K_{1}$, and the proof of the lemma is complete.

Lemma 4. Suppose the conditions in the hypothesis of Lemma 3 hold. Then the sequence $\left\{f\left(x, u^{n}\right)\right\}_{n=1}^{\infty}$ is uniformly absolutely continuous.

To be precise, what we mean by uniformly absolutely continuous is the following: Given $\varepsilon>0$ there exists a $\delta>0$ such that if $E \subset \Omega$ with $\mu(E)<\delta$, then $\int_{E}\left|f\left(x, u^{n}\right)\right| d x<\varepsilon$ for $n=1,2, \ldots$, where $\mu$ is $N$-dimensional Lebesgue measure.

First of all we choose $r>0$ so that

$$
K^{*} / r<\varepsilon / 2,
$$

where $K^{*}$ is the constant in Lemma 3. Next, using (f-2), we choose $\zeta_{r} \in L^{2}(\Omega)$ such that

$$
|f(x, z)| \leqslant \zeta_{r}(x) \text { for a.e. } x \in \Omega \text { and }|z| \leqslant r .
$$

Also we set

$$
A_{n}=\left\{x \in \Omega:\left|u^{n}(x)\right| \leqslant r\right\}, \quad B_{n}=\left\{x \in \Omega:\left|u^{n}(x)\right|>r\right\},
$$

and choose $\delta>0$ such that

$$
\mu(E)<\delta \Rightarrow \int_{E} \zeta_{r}(x) d x<\varepsilon / 2 .
$$

Now suppose $\mu(E)<\delta$ with $\delta$ as in (2.33). Then it follows from Lemma 3 and (2.31)-(2.33) that

$$
\begin{aligned}
\int_{E}\left|f\left(x, u^{n}(x)\right)\right| d x & \leqslant \int_{E \cap A_{n}} \zeta_{r}(x) d x+r^{-1} \int_{E \cap B_{n}}\left|u^{n}(x) f\left(x, u^{n}(x)\right)\right| d x \\
& \leqslant \varepsilon / 2+K^{*} / r
\end{aligned}
$$

for $n=1,2, \ldots$ From (2.30), we see that the right-hand side of this last established inequality is $<\varepsilon$. Consequently $\left\{f\left(x, u^{n}\right)\right\}_{n=1}^{\infty}$ is uniformly absolutely continuous, and the proof of the lemma is complete.

3. Proof of Theorem 1. Since $f$ satisfies (f-1)-(f-3), we see that for every $\varepsilon>0$, there exist a nonnegative function $F_{\varepsilon}(x) \in L^{2}(\Omega)$ such that

$$
z f(x, z) \leqslant \varepsilon z^{2}+F_{\varepsilon}(x)|z| \text { for a.e. } x \in \Omega \text { and } \forall z \in \mathbf{R} .
$$


Consequently, it follows from Lemma 2 that there is a sequence $\left\{u^{n}\right\}_{n=1}^{\infty}$ with the following properties:

$$
\begin{aligned}
u^{n} & =\gamma_{1}^{n} \psi_{1}+\cdots+\gamma_{n}^{n} \psi_{n} \\
\left\langle D_{i} \psi_{k}, a^{i j}\left(\cdot, u^{n}\right) D_{j} u^{n}\right\rangle_{0} & +\left\langle\psi_{k}, b^{j}\left(\cdot, u^{n}, D u^{n}\right) D_{j} u^{n}\right\rangle_{0}+\left\langle\psi_{k}, u^{n}\right\rangle_{0} n^{-1} \\
& =\left\langle\psi_{k}, f\left(\cdot, u^{n}\right)\right\rangle_{0}
\end{aligned}
$$

for $k=1, \ldots, n$, where $\left\{\psi_{k}\right\}_{k=1}^{\infty}$ is the orthonormal sequence with properties (2.1) and (2.2).

With $\|w\|_{0}$ for $w \in L^{2}(\Omega)$ given by (2.22), we define $\|v\|_{1}$ for $v \in W^{1,2}(\Omega)$ by

$$
\|v\|_{1}^{2}=\|v\|_{0}^{2}+\|D v\|_{0}^{2},
$$

where $|D v|^{2}=\left|D_{1} v\right|^{2}+\cdots+\left|D_{N} v\right|^{2}$.

We claim there is a constant $K$ such that

$$
\left\|u^{n}\right\|_{1} \leqslant K \text { for } n=1,2, \ldots
$$

Suppose that (3.5) is false. Then (see [1, p. 169]) there is a subsequence of $\left\{u^{n}\right\}_{n=1}^{\infty}$, which for ease of notation we shall take to be the full sequence, with the following properties:

$$
\lim _{n \rightarrow \infty}\left\|u^{n}\right\|_{1}=\infty
$$

$$
\begin{aligned}
& \text { there is a } v \in W^{1,2}(\Omega) \text { such that } \lim _{n \rightarrow \infty}\left\|v^{n}-v\right\|_{0}=0, \\
& \text { where } v^{n}=u^{n} /\left\|u^{n}\right\|_{1} \text {; }
\end{aligned}
$$

$$
\lim _{n \rightarrow \infty} v^{n}(x)=v(x) \text { a.e. } x \in \Omega ;
$$

$$
\lim _{n \rightarrow \infty} \int_{\Omega} \phi D_{j} v^{n} d x=\int_{\Omega} \phi D_{j} v d x \quad \forall \phi \in C^{\infty}(\Omega), j=1, \ldots, N .
$$

Next, we observe that $D_{j}\left(u^{n}\right)^{2}=2 u^{n} D_{j} u^{n}$ and consequently obtain from (Q-6) that

$$
\left\langle u^{n}, b^{j}\left(\cdot, u^{n}, D u^{n}\right) D_{j} u^{n}\right\rangle_{0}=0 .
$$

Using this fact in conjunction with (3.2), (3.3), and (3.7) gives us that

$$
\left\langle D_{i} v^{n}, a^{i j}\left(\cdot, u^{n}\right) D_{j} v^{n}\right\rangle_{0}+\left\langle v^{n}, v^{n}\right\rangle_{0} n^{-1}=\left\langle u^{n}, f\left(\cdot, u^{n}\right)\right\rangle_{0}\left\|u^{n}\right\|_{1}^{-2} .
$$

Now it follows from (Q-4) that

$$
a^{i j}\left(x, u^{n}\right) D_{i} v^{n} D_{j} v^{n} \geqslant \eta_{0}\left|D v^{n}\right|^{2}
$$

for a.e. $x \in \Omega$, where $\eta_{0}$ is a positive constant. Consequently we obtain from (3.1) and (3.10) that for every $\varepsilon>0$, there is an $F_{\varepsilon} \in L^{2}(\Omega)$ such that

$$
\eta_{0}\left\|D v^{n}\right\|_{0}^{2} \leqslant \varepsilon\left\|v^{n}\right\|_{0}^{2}+\left\|u^{n}\right\|_{1}^{-1}\left\|v^{n}\right\|_{0}\left\|F_{\varepsilon}\right\|_{0} .
$$

From (3.4) and (3.7), we see that $\left\|v_{n}\right\|_{1}^{2}=1$ and furthermore that $\left\|v^{n}\right\|_{0}^{2} \leqslant 1$. We conclude from this fact, in conjunction with (3.6) and (3.11), that $\lim \sup _{n \rightarrow \infty}\|D v\|_{0}^{2}$ $\leqslant \varepsilon \eta_{0}^{-1}$, and therefore, since $\varepsilon>0$ is arbitrary,

$$
\lim _{n \rightarrow \infty}\left\|D v^{n}\right\|_{0}=0 \text {. }
$$


Since $\left|D_{j} v^{n}\right| \leqslant\left|D v^{n}\right|$ for $j=1, \ldots, N$, we obtain from (3.9) and (3.12) that

$$
\int_{\Omega} \phi D_{j} v d x=0 \quad \forall \phi \in C^{\infty}(\Omega) \text { and } j=1, \ldots, N .
$$

Consequently

$$
D_{j} v=0 \text { for } j=1, \ldots, N
$$

and $v$ is equal to a constant a.e. in $\Omega$. To calculate this constant, we see from (3.7) that $\left\|v^{n}\right\|_{1}^{2}=1$. Now $\left\|v^{n}\right\|_{1}^{2}=\left\|v^{n}\right\|_{0}^{2}+\left\|D v^{n}\right\|_{0}^{2}$ and from (3.7), we see that $\left\|v^{n}\right\|_{0}^{2} \rightarrow$ $\|v\|_{0}^{2}$. Therefore it follows from (3.12) that $\lim _{n \rightarrow \infty}\left\|v^{n}\right\|_{1}^{2}=\|v\|_{0}^{2}$ and we conclude from (3.7) that $\|v\|_{0}^{2}=1$. Therefore since $v$ is constant a.e. in $\Omega, v=(2 \pi)^{-N / 2}$ a.e. in $\Omega$ or $v=-(2 \pi)^{-N / 2}$ a.e. in $\Omega$.

We shall suppose

$$
v=(2 \pi)^{-N / 2} \text { a.e. in } \Omega
$$

and arrive at a contradiction. A similar line of reasoning prevails in case the other alternative holds, and we leave the details of this part to the reader.

To arrive at a contradiction, we see from (Q-4) in conjunction with (3.10) that

$$
\left\langle u^{n}, f\left(\cdot, u^{n}\right)\right\rangle_{0} \geqslant 0 \text {. }
$$

Also, from (3.1) with $\varepsilon=1$, we see that

$$
\left(v^{n}\right)^{2}+F_{1}(x) v^{n}\left\|u^{n}\right\|_{1}^{-1}-u^{n} f\left(x, u^{n}\right)\left\|u^{n}\right\|_{1}^{-2} \geqslant 0 \quad \text { a.e. in } \Omega, n=1,2, \ldots
$$

We set

$$
g_{n}=\text { the left-hand side of the inequality in (3.16) }
$$

and obtain from (3.15) that

$$
\int_{\Omega} g_{n}(x) d x \leqslant\left\|v^{n}\right\|_{0}^{2}+\left\|u^{n}\right\|_{1}^{-1} \int_{\Omega}\left|F_{1}(x) v^{n}\right| d x .
$$

From (3.6), (3.7), (3.14), and the fact that $F_{1}(x) \in L^{2}(\Omega)$, we obtain from (3.18) that

$$
\liminf _{n \rightarrow \infty} \int_{\Omega} g_{n}(x) d x \leqslant 1
$$

On the other hand from (3.16), (3.17), Fatou's lemma [7, p. 24] and this last inequality, we obtain that

$$
\int_{\Omega} \liminf g_{n}(x) d x \leqslant 1 .
$$

From (3.7), we see that $u^{n}(x)=v^{n}(x)\left\|u^{n}\right\|$. Therefore it follows from (3.6), (3.8), and (3.14) that

$$
\lim _{n \rightarrow \infty} v^{n}(x)=(2 \pi)^{-N / 2} \text { and } \lim _{n \rightarrow \infty} u^{n}(x)=+\infty \quad \text { a.e. } \in \Omega .
$$

Also, we see that

$$
u^{n} f\left(x, u^{n}\right)\left\|u^{n}\right\|_{1}^{-2}=\left(v^{n}\right)^{2} f\left(x, u^{n}\right) / u^{n} .
$$

Consequently, it follows from (3.6), (3.16), (3.17), (3.20) and (1.3) that

$$
\liminf _{n \rightarrow \infty} g_{n}(x)=(2 \pi)^{-N}-(2 \pi)^{-N} \mathscr{F}_{+}(x) \text { a.e. in } \Omega \text {. }
$$


But then we see from (3.19) that $1-(2 \pi)^{-N} \int_{\Omega} \mathscr{F}_{+}(x) d x \leqslant 1$ and therefore that $\int_{\Omega} \mathscr{F}_{+}(x) d x \geqslant 0$. This fact is contrary to the first inequality in condition (i) in the hypothesis of the theorem. We have arrived at a contradiction and conclude that (3.5) is indeed true.

From the fact that (3.5) holds, it follows (see [1, p. 169]) that there is a subsequence of $\left\{u^{n}\right\}_{n=1}^{\infty}$, which for ease of notation we take to the full sequence, with the following properties:

$$
\begin{aligned}
& \text { there is a } u \in W^{1,2}(\Omega) \text { such that } \lim _{n \rightarrow \infty}\left\|u^{n}-u\right\|_{0}=0 ; \\
& \qquad \lim _{n \rightarrow \infty} u^{n}(x)=u(x) \quad \text { for a.e. } x \in \Omega ; \\
& \lim _{n \rightarrow \infty} \int_{\Omega} w D_{j} u^{n} d x=\int_{\Omega} w D_{j} u d x \quad \forall w \in L^{2}(\Omega), j=1, \ldots, N .
\end{aligned}
$$

From (3.22) and (Q-1), we see that

$$
\lim _{n \rightarrow \infty} a^{i j}\left[x, u^{n}(x)\right]=a^{i j}[x, u(x)] \quad \text { a.e. } x \in \Omega
$$

for $i, j=1, \ldots, N$. It consequently follows from (Q-2) and this last fact that

$$
\lim _{n \rightarrow \infty}\left\|a^{i j}\left(\cdot, u^{n}\right)-a^{i j}(\cdot, u)\right\|_{0}=0 .
$$

But this fact, in turn, in conjunction with (3.5) and the Schwarz inequality implies that for fixed $k$

$$
\lim _{n \rightarrow \infty}\left\langle D_{i} \psi_{k},\left[a^{i j}\left(\cdot, u^{n}\right)-a^{i j}(\cdot, u)\right] D_{j} u^{n}\right\rangle_{0}=0 .
$$

From (3.23), in conjunction with (Q-2), we also see that

$$
\lim _{n \rightarrow \infty}\left\langle D_{i} \psi_{k}, a^{i j}(\cdot, u) D_{j} u^{n}\right\rangle_{0}=\left\langle D_{i} \psi_{k}, a^{i j}(\cdot, u) D_{j} u\right\rangle_{0} .
$$

We conclude from this last fact in conjunction with (3.24) that

$$
\lim _{n \rightarrow \infty}\left\langle D_{i} \psi_{k}, a^{i j}\left(\cdot, u^{n}\right) D_{j} u^{n}\right\rangle_{0}=\left\langle D_{i} \psi_{k}, a^{i j}(\cdot, u) D_{j} u\right\rangle_{0}
$$

for $k=1,2, \ldots$.

Next, from (Q-6), we see that

$$
\int_{\Omega} b^{j}\left(\cdot, u^{n}, D u^{n}\right) D_{j}\left(u^{n} \psi_{k}\right) d x=0
$$

and consequently that

$$
\left\langle\psi_{k}, b^{j}\left(\cdot, u^{n}, D u^{n}\right) D_{j} u^{n}\right\rangle_{0}=-\left\langle u^{n}, b^{j}\left(\cdot, u^{n}, D u^{n}\right) D_{j} \psi_{k}\right\rangle_{0} .
$$

On the other hand, from (Q-5) we see that

$$
\int_{\Omega}\left|u^{n}-u\left\|b^{j}\left(x, u^{n}, D u^{n}\right) \mid d x \leqslant\right\| u^{n}-u \|_{0}\left[\|b(x)\|_{0}+\eta_{1}\left\|u^{n}\right\|_{0}+\eta_{2}\left\|D u^{n}\right\|_{0}\right] .\right.
$$

From (3.5) and (3.21), we also see that the right-hand side of this last inequality goes to 0 as $n \rightarrow \infty$, and consequently from (3.26), we obtain that

$$
\lim _{n \rightarrow \infty}\left\langle\psi_{k}, b^{j}\left(\cdot, u^{n}, D u^{n}\right) D_{j} u^{n}\right\rangle_{0}=-\lim _{n \rightarrow \infty}\left\langle u, b^{j}\left(\cdot, u^{n}, D u^{n}\right) D_{j} \psi_{k}\right\rangle_{0} .
$$


However, from (3.23), we see that $\left\{D u^{n}\right\}_{n=1}^{\infty}$ is a sequence of vector-valued functions in $\left\{L^{2}(\Omega)\right\}^{N}$ which tends weakly to $D u \in\left[L^{2}(\Omega)\right]^{N}$. Also from (3.21), we see that $\left\{u^{n}\right\}_{n=1}^{\infty}$ tends strongly to $u$ in $L^{2}(\Omega)$. Therefore, it follows from (Q-7) that

$$
\lim _{n \rightarrow \infty}\left\langle u, b^{j}\left(\cdot, u^{n}, D u^{n}\right) D_{j} \psi_{k}\right\rangle_{0}=\left\langle u, b^{j}(\cdot, u, D u) D_{j} \psi_{k}\right\rangle_{0} .
$$

But, since $u \in W^{1,2}(\Omega)$, we see as earlier,

$$
\left\langle u, b^{j}(\cdot, u, D u) D_{j} \psi_{k}\right\rangle_{0}=-\left\langle\psi_{k}, b^{j}(\cdot, u, D u) D_{j} u\right\rangle_{0} .
$$

We consequently conclude from these last two equalities and (3.27) that

$$
\lim _{n \rightarrow \infty}\left\langle\psi_{k}, b^{j}\left(\cdot, u^{n}, D u^{n}\right) D_{j} u^{n}\right\rangle_{0}=\left\langle\psi_{k}, b^{j}(\cdot, u, D u) D_{j} u\right\rangle_{0}
$$

for $k=1,2, \ldots$

Next, from (3.5) and Lemma 4, we see that the sequence $\left\{f\left(x, u^{n}\right)\right\}_{n=1}^{\infty}$ is uniformly absolutely continuous. Also, we see from (f-1) and (3.22) that $\lim _{n \rightarrow \infty} f\left[x, u^{n}(x)\right]=f[x, u(x)]$ for a.e. $x \in \Omega$. We conclude from Egoroff's theorem $[\bar{T}$, p. 75$]$ and from Fatou's lemma that

$$
f(x, u) \in L^{1}(\Omega)
$$

and

$$
\lim _{n \rightarrow \infty} \int_{\Omega} \psi_{k}(x) f\left(x, u^{n}\right) d x=\int_{\Omega} \psi_{k}(x) f(x, u) d x
$$

for $k=1,2, \ldots$

From (3.3), (3.21), (3.25), (3.28), and (3.30), we see that

$$
\left\langle D_{i} \psi_{k}, a^{i j}(\cdot, u) D_{j} u\right\rangle_{0}+\left\langle\psi_{k}, b^{j}(\cdot, u, D u) D_{j} u\right\rangle_{0}=\left\langle\psi_{k}, f(\cdot, u)\right\rangle_{0}
$$

for $k=1,2, \ldots$.

From (Q-2), we see that $a^{i j}(\cdot, u) D_{j} u \in L^{1}(\Omega)$. From $(\mathrm{Q}-5)$, we see that $b^{j}(\cdot, u, D u) D_{j} u \in L^{1}(\Omega)$. From (3.29), we have that $f(x, u) \in L^{1}(\Omega)$. Also, it follows from (2.2) that given $\phi \in C^{\infty}(\Omega)$, there is a sequence $\left\{\phi_{n}\right\}_{n=1}^{\infty}$ and real constants $\left\{c_{q}^{n}\right\}_{q=1}^{n}$ such that $\phi_{n}=c_{1}^{n} \psi_{1}+\cdots+c_{n}^{n} \psi_{n}, \lim _{n \rightarrow \infty} \phi_{n}(x)=\phi(x)$, and $\lim _{n \rightarrow \infty} D_{j} \phi_{n}(x)=D_{j} \phi(z)$ uniformly for $x \in \Omega, j=1, \ldots, N$. We conclude first that (3.31) holds with $\psi_{k}$ replaced by $\phi_{n}$, and next on passing to the limit as $n \rightarrow \infty$, that

$$
\left\langle D_{i} \phi, a^{i j}(\cdot, u) D_{j} u\right\rangle_{0}+\left\langle\phi, b^{j}(\cdot, u, D u) D_{j} u\right\rangle_{0}=\langle\phi, f(\cdot, u)\rangle_{0} .
$$

But this is $(1.3)^{\prime}$. From (3.21) we see that $u \in W^{1,2}(\Omega)$. We conclude that $u$ is indeed a distribution solution of $Q u=f(x, u)$.

To complete the proof of the theorem, it remains to show that $u f(x, u) \in L^{1}(\Omega)$. From (3.22) and (f-1), it follows that

$$
\lim _{n \rightarrow \infty} u^{n}(x) f\left[x, u^{n}(x)\right]=u(x) f[x, u(x)] \quad \text { a.e. } \in \Omega .
$$

On the other hand from (3.5) and Lemma 3, we see that there is a constant $K^{*}$ such that $\int_{\Omega}\left|u^{n}(x) \| f\left[x, u^{n}(x)\right]\right| d x \leqslant K^{*}$. Consequently, it follows from Fatou's lemma and (3.32) that $\int_{\Omega}|u(x)||f(x, u(x))| d x \leqslant K^{*}$, and the proof of Theorem 1 is complete. 
4. Proof of Theorem 2. We first establish the necessary condition of the theorem. Suppose then that (1.8) and (1.9) hold, where $u \in W^{1,2}(\Omega)$. We choose $\phi \equiv 1$ in (1.8) and observe that

$$
\int_{\Omega} a^{i j}(x, u) D_{j} u D_{i} 1 d x=0 .
$$

Also, we see from (Q-6) on choosing $v=u$ that

$$
\int_{\Omega} b^{j}(x, u, D u) D_{j} u d x=0 .
$$

We consequently conclude from these last two equalities and the fact that (1.8) holds that

$$
\int_{\Omega} g(u) d x=h(1) .
$$

Using the first inequality in (1.9) in conjunction with (4.1) gives us the first inequality in (1.10). Using the second inequality in (1.9) in conjunction with (4.1) gives us the second inequality in (1.10), and the proof of the necessary condition in Theorem 2 is complete.

To establish the sufficient condition in the theorem, we first observe that the following lemma holds.

Lemma 5. Let $g$ be in $C(\mathbf{R}) \cap L^{\infty}(\mathbf{R})$ and $h \in W^{-1,2}(\Omega)$. Suppose that $Q$ satisfies (Q-1)-(Q-6). Then if $n$ is a given positive integer, there is a function $u^{n}=\gamma_{1}^{n} \psi_{1}$ $+\cdots+\gamma_{n}^{n} \psi_{n}$ such that

$$
\begin{gathered}
\int_{\Omega}\left[a^{i j}\left(x, u^{n}\right) D_{j} u^{n} D_{i} \psi_{k}+\psi_{k} b^{j}\left(x, u^{n}, D u^{n}\right) D_{j} u^{n}+u^{n} \psi_{k} n^{-1}\right] d x \\
=\int_{\Omega} \psi_{k}(x) g\left(u^{n}\right) d x-h\left(\psi_{k}\right) \quad \text { for } k=1, \ldots, n .
\end{gathered}
$$

The proof of the above lemma is exactly the same as the proof of Lemma 1 except $S_{k}(\alpha)$ in (2.11) now becomes $S_{k}(\alpha)=\left\langle\psi_{k}, g\left(\alpha_{q} \psi_{q}\right)\right\rangle_{0}-h\left(\psi_{k}\right)$ for $k=1, \ldots, n$, where the summation convention is used for $q=1, \ldots, n$. The reader will have no difficulty in filling in the details.

We now proceed with the proof of the sufficiency. We observe that the conditions in the hypothesis of Theorem 2 imply those in the hypothesis of Lemma 5. So for each positive integer $n$, we let $u^{n}$ designate the solution satisfying (4.2). We claim, as in the proof of Theorem 1, that there is a constant $K$ such that

$$
\left\|u^{n}\right\|_{1} \leqslant K \text { for } n=1,2, \ldots,
$$

where $\|\cdot\|_{1}$ is defined in (3.4).

Suppose that (4.3) is false. Then (see [1, p. 169]) there is a subsequence of $\left\{u^{n}\right\}$, which for ease of notation we shall take to be the full sequence, which satisfies (3.6)-(3.9) and furthermore, since $h \in W^{-1,2}(\Omega)\left(\equiv W^{1,2}(\Omega)^{*}\right)$, satisfies

$$
\lim _{n \rightarrow \infty} h\left(v^{n}\right)=h(v)
$$

where $v^{n}=u^{n} /\left\|u^{n}\right\|_{1}$ and $v=u /\|u\|_{1}$. 
Now, as the proof of Theorem $1,\left\langle u^{n}, b^{j}\left(\cdot, u^{n}, D u^{n}\right) D_{j} u^{n}\right\rangle_{0}=0$; so we obtain from (4.2) that

$$
\left\langle D_{i} v^{n}, a^{i j}\left(\cdot, u^{n}\right) D_{j} v^{n}\right\rangle+\left\langle v^{n}, v^{n}\right\rangle_{0} n^{-1}=\left[\left\langle u^{n}, g\left(u^{n}\right)\right\rangle_{0}-h\left(u^{n}\right)\right]\left\|u^{n}\right\|_{1}^{-2} \text {. }
$$

From (Q-4), we see that the left-hand side of (4.5) majorizes $\eta_{0} \mid\left\|D v^{n}\right\| \|_{0}^{2}$. On the other hand, since $g \in L^{\infty}(\mathbf{R})$ and $h \in W^{-1,2}(\Omega)$, we see that the right-hand side of (4.5) is majorized by $K_{1}\left\|u^{n}\right\|_{1}\left\|u^{n}\right\|_{1}^{-2}$, where $K_{1}$ is a constant independent of $n$. We conclude from (4.5) that

$$
\left\|D v^{n}\right\|_{0}^{2} \leqslant \eta_{0}^{-1} K_{1}\left\|u^{n}\right\|_{1}^{-1} \text { for } n=1,2, \ldots
$$

From (3.6) and (4.6), we immediately obtain that

$$
\lim _{n \rightarrow \infty}\left\|D v^{n}\right\|_{0}^{2}=0 \text {. }
$$

From (3.7), we see that $\left\|v^{n}\right\|_{1}^{2}=1$ and consequently from (3.4), (3.7), and (4.7) that

$$
\|v\|_{0}^{2}=\lim _{n \rightarrow \infty}\left\|v^{n}\right\|_{0}^{2}=1
$$

Also, from (3.9) and (4.7), we see that

$$
D_{j} v=0, \quad j=1, \ldots, N \text {. }
$$

Now by (3.7), $v \in W^{1,2}(\Omega)$. It follows, therefore, from (4.8) and (4.9) that $v=(2 \pi)^{-N / 2}$ a.e. in $\Omega$ or $v=-(2 \pi)^{-N / 2}$ a.e. in $\Omega$.

We shall suppose

$$
v=(2 \pi)^{-N / 2} \text { a.e. in } \Omega,
$$

and arrive at a contradiction. As the reader will easily see, a similar line of reasoning gives a contradiction in case the other alternative holds.

Suppose then that (4.10) holds. We observe from (Q-4) and (4.5) that $0 \leqslant$ $\left\langle u^{n}, g\left(u^{n}\right)\right\rangle-h\left(u^{n}\right)$, and consequently, using the linearity of $h$, that

$$
h\left(v^{n}\right) \leqslant\left\langle v^{n}, g\left(u^{n}\right)\right\rangle_{0} .
$$

Since $u^{n}=\left\|u_{n}\right\|_{1} v^{n}$, we see from (3.6), (3.8), and (4.10) that

$$
\lim _{n \rightarrow \infty} g\left[u^{n}(x)\right] v^{n}(x)=(2 \pi)^{-N / 2} g(\infty) \text { a.e. in } \Omega \text {. }
$$

Also, since $g \in L^{\infty}(\mathbf{R})$, it follows from (4.8) that there exists a constant $K_{2}$ such that

$$
\int_{\Omega}\left|g\left(u^{n}\right) v^{n}\right|^{2} d x \leqslant K_{2} \quad \text { for } n=1,2, \ldots
$$

From this last fact and Schwarz' inequality, it follows that the sequence $\left\{g\left(u^{n}\right) v^{n}\right\}_{n=1}^{\infty}$ is uniformly absolutely continuous and consequently from (4.12) and Egoroff's Theorem [7, p. 75],

$$
\lim _{n \rightarrow \infty}\left\langle v^{n}, g\left(u^{n}\right)\right\rangle_{0}=(2 \pi)^{N / 2} g(\infty) .
$$

On the other hand from (4.4), (4.10), and the linearity of $h$, we see that

$$
\lim _{n \rightarrow \infty} h\left(v^{n}\right)=(2 \pi)^{-N / 2} h(1) \text {. }
$$


But then we obtain from (4.11), (4.13), and this last inequality that $h(1) \leqslant$ $(2 \pi)^{N} g(\infty)$. This fact is a contradiction to the first equality in (1.10). We conclude that (4.3) does indeed hold.

From the fact that (4.3) holds, we see from [1, p. 169] that there is a subsequence of $\left\{u^{n}\right\}$, which for ease of notation we shall take to be the full sequence, and a function $u \in W^{1,2}(\Omega)$ which satisfies (3.21), (3.22), and (3.23).

Using (Q-1), (Q-2), (3.21), (3.22), and (4.3) (which is the analogue of (3.5)), we obtain exactly as in the proof of Theorem 1 that

$$
\lim _{n \rightarrow \infty}\left\langle D_{i} \psi_{k}, a^{i j}\left(\cdot, u^{n}\right) D_{j} u^{n}\right\rangle_{0}=\left\langle D_{i} \psi_{k}, a^{i j}(\cdot, u) D_{j} u\right\rangle_{0}
$$

for $k=1,2, \ldots$ In a similar manner, we obtain from the proof of Theorem 1 that

$$
\lim _{n \rightarrow \infty}\left\langle\psi_{k}, b^{j}\left(\cdot, u^{n}, D u^{n}\right) D_{j} u^{n}\right\rangle_{0}=\left\langle\psi_{k}, b^{j}(\cdot, u, D u) D_{j} u\right\rangle_{0} .
$$

Next, from (3.22) and the fact that $g \in C(\mathbf{R})$, we see that

$$
\lim _{n \rightarrow \infty} g\left[u^{n}(x)\right]=g[u(x)] \text { for a.e. } x \in \Omega \text {. }
$$

Since $g$ is also in $L^{\infty}(\mathbf{R})$, it follows from this last equality and the Lebesgue dominated convergence theorem that

$$
\lim _{n \rightarrow \infty}\left\langle\psi_{k}, g\left(u^{n}\right)\right\rangle_{0}=\left\langle\psi_{k}, g(u)\right\rangle_{0} \text { for } k=1,2, \ldots
$$

From (3.21), (4.2), (4.14), (4.15), and (4.16), we finally conclude that

$$
\left\langle D_{i} \psi_{k}, a^{i j}(\cdot, u) D_{j} u\right\rangle_{0}+\left\langle\psi_{k}, b^{j}(\cdot, u, D u) D_{j} u\right\rangle_{0}=\left\langle\psi_{k}, g(u)\right\rangle_{0}-h\left(\psi_{k}\right)
$$

for $k=1,2, \ldots$.

Next, given $\phi \in C^{\infty}(\Omega)$, we see from (2.2) that there exists a set of real constants $\left\{c_{q}^{n}\right\}_{q=1}^{n}$ and a sequence $\left\{\phi_{n}\right\}_{n=1}^{\infty}$ with

$$
\phi_{n}=c_{1}^{n} \psi_{1}+\cdots+c_{n}^{n} \psi_{n}
$$

such that both of the following prevail:

$$
\lim _{n \rightarrow \infty} \phi_{n}(x)=\phi(x) \quad \text { uniformly for } x \in \Omega,
$$

$$
\lim _{n \rightarrow \infty} D_{j} \phi_{n}(x)=D_{j} \phi(x) \quad \text { uniformly for } x \in \Omega, j=1, \ldots, N \text {. }
$$

These last two facts, in particular, imply that $\lim _{n \rightarrow \infty}\left\|\phi_{n}-\phi\right\|_{1}=0$, and consequently since $h \in W^{-1,2}(\Omega)$ that

$$
\lim _{n \rightarrow \infty} h\left(\phi_{n}\right)=h(\phi) .
$$

Since $u \in W^{1,2}(\Omega)$, it follows from (Q-2) that

$$
a^{i j}(x, u) D_{j} u \in L^{1}(\Omega) \text { for } i=1, \ldots, N .
$$

Likewise, it follows from (Q-5) that

$$
b^{j}(x, u, D u) D_{j} u \in L^{1}(\Omega) .
$$


From (4.20) and (4.22), we obtain

$$
\lim _{n \rightarrow \infty}\left\langle D_{j} \phi_{n}, a^{i j}(\cdot, u) D_{j} u\right\rangle_{0}=\left\langle D_{j} \phi, a^{i j}(\cdot, u) D_{j} u\right\rangle_{0} .
$$

Likewise from (4.19) and (4.23), we obtain

$$
\lim _{n \rightarrow \infty}\left\langle\phi_{n}, b^{j}(\cdot, u, D u) D_{j} u\right\rangle_{0}=\left\langle\phi, b^{j}(\cdot, u, D u) D_{j} u\right\rangle_{0} .
$$

Also, we see from (4.19) that

$$
\lim _{n \rightarrow \infty}\left\langle\phi_{n}, g(u)\right\rangle_{0}=\langle\phi, g(u)\rangle_{0} .
$$

We are now ready to conclude the proof of the theorem. From (4.18) we see that (4.17) holds with $\psi_{k}$ replaced by $\phi_{n}$. But then we obtain from (4.21), (4.24), (4.25), and (4.26) on passing to the limit as $n \rightarrow \infty$ that (4.17) holds with $\psi_{k}$ replaced by $\phi$, i.e.,

$$
\left\langle D_{i} \phi, a^{i j}(\cdot, u) D_{j} u\right\rangle_{0}+\left\langle\phi, b^{j}(\cdot, u, D u) D_{j} u\right\rangle=\langle\phi, g(u)\rangle_{0}-h(\phi)
$$

$\phi$, however, is an arbitrary given function in $C^{\infty}(\Omega)$, so (4.27) shows that (1.8) is indeed true, and the proof of Theorem 2 is complete.

\section{REFERENCES}

1. L. Bers, F. John and M. Schecter, Partial differential equations, Lectures in Appl. Math., vol. 3, Interscience, New York, 1964.

2. H. Brezis and L. Nirenberg, Characterization of the ranges of some nonlinear operators and applications to boundary value problems, Ann. Scuola Norm. Sup. Pisa 5 (1978), 225-326.

3. D. G. de Figueiredo and J. P. Gossez, Nonlinear partial differential equations near the first eigenvalue,

J. Differential Equations 30 (1978), 1-19.

4. P. Hess, On a theorem by Landesman and Lazer, Indiana Univ. Math. J. 23 (1974), 827-829.

5. J. L. Kazdan and F. W. Warner, Remarks on some quasilinear elliptic equations, Comm. Pure Appl. Math. 28 (1975), 567-597.

6. E. M. Landesman and A. C. Lazer, Nonlinear perturbations of linear elliptic boundary value problems at resonance, J. Math. Mech. 19 (1970), 609-623.

7. W. Rudin, Real and complex analysis (2nd ed.), McGraw-Hill, New York, 1974.

Department of Mathematics, University of California, Riverside, California 92521 